\title{
Genome-wide association study of individual differences of human lymphocyte profiles using large-scale cytometry data
}

\author{
Daigo Okada' ${ }^{1}$ Naotoshi Nakamura ${ }^{1} \cdot K^{2}$ azuya Setoh ${ }^{2} \cdot$ Takahisa Kawaguchi $^{2} \cdot$ Koichiro Higasa $^{2,3} \cdot$ \\ Yasuharu Tabara ${ }^{2} \cdot$ Fumihiko Matsuda $^{2} \cdot$ Ryo Yamada $^{1}$
}

Received: 18 August 2020 / Revised: 26 October 2020 / Accepted: 31 October 2020 / Published online: 23 November 2020

(c) The Author(s) 2020. This article is published with open access

\begin{abstract}
Human immune systems are very complex, and the basis for individual differences in immune phenotypes is largely unclear. One reason is that the phenotype of the immune system is so complex that it is very difficult to describe its features and quantify differences between samples. To identify the genetic factors that cause individual differences in whole lymphocyte profiles and their changes after vaccination without having to rely on biological assumptions, we performed a genome-wide association study (GWAS), using cytometry data. Here, we applied computational analysis to the cytometry data of 301 people before receiving an influenza vaccine, and 1, 7, and 90 days after the vaccination to extract the feature statistics of the lymphocyte profiles in a nonparametric and data-driven manner. We analyzed two types of cytometry data: measurements of six markers for B cell classification and seven markers for $\mathrm{T}$ cell classification. The coordinate values calculated by this method can be treated as feature statistics of the lymphocyte profile. Next, we examined the genetic basis of individual differences in human immune phenotypes with a GWAS for the feature statistics, and we newly identified seven significant and 36 suggestive single-nucleotide polymorphisms associated with the individual differences in lymphocyte profiles and their change after vaccination. This study provides a new workflow for performing combined analyses of cytometry data and other types of genomics data.
\end{abstract}

\section{Introduction}

The human immune system is highly complex [1]. It is still unclear what individual differences exist in the phenotype of a healthy person's immune system. Also, it is not clear how the immune system phenotype changes with the immune response. One reason is that the phenotype of the

Supplementary information The online version of this article (https:// doi.org/10.1038/s10038-020-00874-x) contains supplementary material, which is available to authorized users.

$\triangle$ Ryo Yamada

ryamada@genome.med.kyoto-u.ac.jp

1 Department of Statistical Genetics, Center for Genomic Medicine, Graduate School of Medicine, Kyoto University, Kyoto, Japan

2 Department of Human Disease Genomics, Center for Genomic Medicine, Graduate School of Medicine, Kyoto University, Kyoto, Japan

3 Department of Genome Analysis, Institute of Biomedical Science, Kansai Medical University, Hirakata, Japan immune system is so complex that it is very difficult to describe its features and quantify differences between samples.

To investigate complex biological phenomena, such as the immune response to vaccination, genome-wide association studies (GWASs) are a powerful approach. They can detect the single-nucleotide polymorphisms (SNPs) that are associated with complex traits [2]. For the immune response to vaccination, several GWAS analyses have been conducted, and in these analyses, the blood cytokine measurement or titer [3,4] has been used to represent the immune response. These previous studies have successfully detected genetic variants associated with the immune response to vaccination. However, the immunophenotype is very complex and difficult to comprehensively characterize by using the concentrations of single blood metabolites.

The immunophenotype is not only a complex trait but is also strongly characterized by the lymphocyte profile, as measured by cytometry data [5]. Recently, large-scale flow cytometry data analyses of the immune response to vaccination have revealed differentially expressed genes before and after vaccination, in addition to crucial subsets of the 
immune response [6-8]. However, these studies have not focused on individual differences, but rather the general mechanism of the immune response to vaccination, and then only on specific lymphocyte subsets based on the previous biological knowledge.

In the field of computational biology, several methods have been proposed to examine the differences of cell population profiles among multiple cytometry samples in a data-driven and nonparametric manner [9-11]. In these approaches, cytometry data are considered as a sample from an unknown multidimensional probability distribution. These methods quantify dissimilarities between probability distributions based on information theory, apply a multidimensional scaling (MDS) method to the distance matrix, and then embed them in a low-dimensional space. The obtained coordinate values can be treated as feature statistics of the cell population profile, which enables sample differences to be visualized in low-dimensional space.

In this study, we used a computational method for largescale cytometry data and embedded the lymphocyte profiles into low-dimensional spaces based on the dissimilarities among samples. The coordinate values calculated by this method can be treated as feature statistics of the lymphocyte profile. To conduct the following analyses, the extraction of some feature statistics from cytometry data is a necessary step, which enables us to examine the correlation with SNP genotype without needing biological assumptions. We identified new SNPs related to the individual differences in lymphocyte profiles and their changes after vaccination via a GWAS for these feature statistics. Our results provide novel insights into the genetics of individual differences of the immune response.

\section{Materials and methods}

\section{Flow cytometry and SNP genotype data}

In this study, we used data that we obtained from a related project with the Nagahama Cohort Study [12]. This project profiled 301 healthy people (103 men and 198 women) aged between 32 and 66 . The participants had received an injection of trivalent inactivated influenza vaccine that contained three types of HA antigens from A/California/7/2009 (H1N1) pdm09, A/Victoria/210/2009 (H3N2), and B/Brisbane/60/ 2008. Peripheral blood was collected at four time points, before influenza vaccine (Day 0) and 1 day (Day 1), 7 days (Day 7), and 90 days (Day 90) after vaccination. Although FACS data were taken at all four time points, a total of 1173 samples were used because of partial loss. Two types of FACS data (B cell FACS and T cell FACS) were obtained for each person at each time point. In B cell FACS, a set of six cell surface markers (CD19, IgM, IgD, CD21, CD27, and
CD138) for B cell classification were measured. These markers can be used to identify plasma cells, immature B cells, naive $\mathrm{B}$ cells, non-switched memory $\mathrm{B}$ cells, class-switched memory B cells, and double-negative memory B cells with conventional gating methods [13-15]. For T cell FACS, a set of seven cell surface markers (CD3, CD4, CD8, CD45RA, CD45RO, CD25, and CCR7) for $\mathrm{T}$ cell classification were measured. CD4 and CD8 can be used to identify helper $\mathrm{T}$ cells, killer $\mathrm{T}$ cells, and double-negative $\mathrm{T}$ cells. CD45RA, CD45RO, and CD25 can be used to identify naive T cells, memory $\mathrm{T}$ cells, and effector $\mathrm{T}$ cells [16-23]. CCR7 is a marker for identifying exhausted $\mathrm{T}$ cells (CCR7 negative) from naive $\mathrm{T}$ cells (CCR7 positive), and for classifying memory $\mathrm{T}$ cells into central memory $\mathrm{T}$ cells and effector memory $\mathrm{T}$ cells. These markers may not be sufficient to accurately classify all B cell and T cell subsets. For example, $\mathrm{CD} 25$ is known to be a marker of regulatory T cells, as well as in the T cell subset described above [24], and plasma cells that do not express CD138 are also present [25]. We selected these marker sets to capture the information of as many lymphocyte subsets as possible with a limited number of markers, rather than for quantification and classification of each subset.

All FACS data were preprocessed with compensation, normalization by inverse hyperbolic function arcsine transformation for each marker, and lymphocyte gating. In treating cytometric data as a probability distribution, pretreatment can be potentially an artifact. Therefore, we decided to perform only minimal pretreatment in our study design. Our lymphocyte gating process selected CD19positive or CD138-positive cells in the case of B cell FACS, and CD3-positive cells in the case of T cell FACS, which extracted the lymphocytes. An example of this lymphocyte gating is shown in Supplementary File S1. The preprocessed data are the same as in the preprint paper of our previous work [11].

We used the SNP genotype data from our previous paper [26]. For the SNP genotype data, 1,665,663 SNP genotypes on the autosomes of 298 people were used, satisfying the minor allele frequency (MAF) $>0.01$ and the Hardy-Weinberg equilibrium test $P$ value of $>1.0 \times 10^{-7}$. Although MAF $>0.05$ was used in ref. [26], in this study we used MAF $>0.01$ so that we included more SNPs in the analysis. The annotation of the SNPs was performed by the web-based tool SNPnexus [27] based on GRCh38 and gene annotation in the Ensembl database.

\section{Comprehensive quantification of cell subset fractions}

First of all, in order to describe how broad lymphocyte subset populations differ over the course of vaccination, we conducted comprehensive quantification of lymphocyte 
subsets, using an automatic approach with a parametric model (detail method in Supplementary File S2). For each marker of each FACS data, a cutoff value for determining positive/negative was calculated. We excluded IgM and CD138 in the B cell FACS dataset from this analysis, and all remaining markers showed a bimodal distribution. CD3 in the T cell FACS dataset was also excluded because we had selected CD3-positive cells in the lymphocyte gating process. Then, the abundance of $2^{4}=16$ subsets in B cell FACS and $2^{6}=64$ subsets in T cell FACS were quantified, using the positive/negative combination of all cells defined by the cutoff values. The percentage of the total number of cells for each subset was calculated to obtain a cell ratio matrix with the number of samples $x$ the number of subsets. At each time point, changes before and after vaccination were tested using a Wilcoxon signed-rank test, and a subset with FDR $Q$ value $<0.01$ was searched.

\section{Embedding lymphocyte profiles into Euclidean space}

In order to conduct a GWAS of the individual differences of human lymphocyte profiles, we obtained the feature statistics using cytometry data in a data-driven and nonparametric manner. The procedure for extracting feature statistics from FACS data and embedding them with multidimensional markers to Euclidean space was as follows (Fig. 1a). First, an equally spaced $m$ grid was set for each marker expression value. $m=10$ and $m=8$ were used in B cell FACS and T cell FACS, respectively. First, we decided the range of each marker. For each sample, we calculated the 5th percentile and 95th percentile of each marker expression, and used the range of each marker between the minimum 5th percentile value and maximum 95th percentile value among all samples. By dividing these ranges into $m$ parts, we decided $m^{n}$ lattice points where $n$ is the number of markers. Discrete approximation of the probability density function of a multidimensional distribution for these lattice points was calculated using the $k$-nearest neighbor method with $k=60$. Normalization was performed so that the sum of each grid was 1 , which is the estimated probability mass function of the FACS data. Next, the square root of the Jensen-Shannon distance [28] between the population distributions was estimated. The square root of the Jensen-Shannon distance is a distance metric between probability distributions. Jensen-Shannon distance is defined by the KL divergence and can be written as follows:

$\mathrm{JS}(p \| q)=\frac{1}{2}\left(\mathrm{KL}\left(p \| \frac{p+q}{2}\right)+\mathrm{KL}\left(q \mid \frac{p+q}{2}\right)\right)$.

As a result, a sample $\times$ sample dissimilarity matrix was constructed. By applying MDS to this dissimilarity matrix, all cytometry data were embedded into the low-dimensional Euclidean space that best reflected their dissimilarity. The number of meaningful MDS coordinates was determined based on the elbow of the eigenvalue plot. In this research, the top $K$ MDS coordinates were defined as the meaningful coordinates:

$K={ }_{i}^{\text {arg } \min }\left(\left|\operatorname{Eig}_{i+1}-\operatorname{Eig}_{i}\right|-\left|\operatorname{Eig}_{i}-\operatorname{Eig}_{i-1}\right|\right)-1$,

where $i$ takes integer values from two to the number of samples -1 , and $\operatorname{Eig}_{i}$ is the $i$ th largest eigenvalue. The selected meaningful MDS coordinates were used for subsequent analysis.

We used these MDS coordinate values as the lymphocyte profile feature statistics. However, the biological significance of these feature statistics is still unclear. Using a cell ratio matrix calculated with the parametric model, we examined which lymphocyte subsets the MDS coordinates explain. For all pairs of MDS coordinates and lymphocyte fractions, we calculated the Kendall's correlation coefficient and $P$ value. We then considered MDS coordinates as traits, and analyzed the association between MDS coordinates and SNP genotype data (Fig. 1b). These analyses identified genetic variations with whole lymphocyte profile differences.

\section{GWAS for the MDS coordinates}

To examine the genetic effects of the lymphocyte profiles, a GWAS was performed on the MDS coordinate values, and SNPs that were significantly related to these feature statistics were examined. Calculations were performed by linear regression for each SNP using the software PLINK v1.07 [29]. The target traits are the coordinate values of MDS1, MDS2, MDS3, MDS4, and MDS5 in B cell FACS, and MDS1 and MDS2 in T cell FACS at each of Days 0, 1, 7, and 90. In the case of Day 0, the following linear regression model was used:

$\mathrm{MDS}_{i}=b_{0}+b_{1} \mathrm{SEX}+b_{2} \mathrm{AGE}+b_{3} \mathrm{SNP}+b_{4}$ GROUP + error

where SNP represents the SNP allele count, SEX and AGE are the covariates, and the error term is the random error under a normal distribution. GROUP is also a covariate that takes one of two groups and represents a batch effect. In the case of Days 1, 7, and 90, we added the MDS coordinates value from Day 0 (BASELINE) as a covariate to the model, and the following linear regression model was used:

$$
\begin{aligned}
\mathrm{MDS}_{i}= & b_{0}+b_{1} \mathrm{SEX}+b_{2} \mathrm{AGE}+b_{3} \mathrm{SNP}+b_{4} \mathrm{GROUP} \\
& +b_{5} \mathrm{BASELINE}+\text { error. }
\end{aligned}
$$

In the case of Days 1, 7, and 90, the following model with the value at Day 0 added as a baseline covariate was 
Fig. 1 Outline of embedding a FACS dataset into a lowdimensional space. a The procedure for extracting feature statistics from FACS data and embedding them with multidimensional markers to Euclidean space is as follows. We estimated the probability distributions of multiple marker expressions of each FACS dataset, the square root of the Jensen-Shannon distance between these distributions was estimated, and a sample $x$ sample dissimilarity matrix was constructed. By applying MDS to this dissimilarity matrix, all data were embedded in the lowdimensional Euclidean space that best reflected the relation between samples in terms of their dissimilarity. b We considered an MDS coordinate value as trait and analyzed the association between MDS coordinates and SNP genotype data (a)

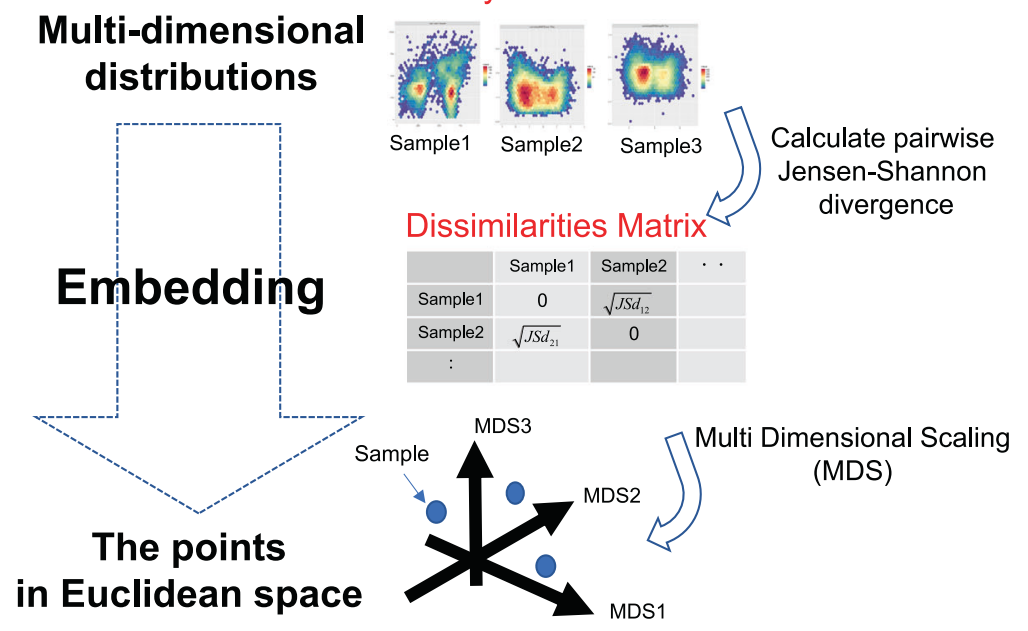

(b)

MDS coordinate values

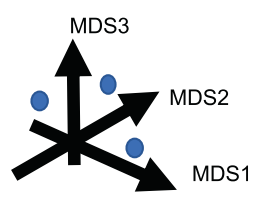

used. $P$ values were calculated for each SNP. Using 50,000 randomly picked SNPs, we visualized the correlation of the regression coefficients as beta and $P$ values between MDS coordinates with the python matplotlib library.

For each day of B cell FACS and T cell FACS, we integrated the $P$ values of MDS coordinates into one representative value using the meta-analysis method. The differences of the lymphocyte profiles were retained by the Euclidean distance on the MDS coordinate system. We considered that the effects of the SNPs are represented as a vector on the MDS coordinate system, which is unlikely to be orthogonal to a particular MDS coordinate. Then, we integrated the $P$ values based on the maximum $P$ values, assuming that the candidate SNPs for differences in lymphocyte profiles were associated with all MDS coordinates at 1-day point. We used the maximump function in the R package "metap" for this procedure [30]. Also, we calculated the genomic inflation factor $(\lambda)$ based on median chi-squared values. When $\lambda$ is almost equal to 1 (for example, $\lambda<1.1$ ), the population structure is considered to be subtle [31]. The results of the GWAS were visualized by a Manhattan plot, which was drawn by the $\mathrm{R}$ package "qqman" [32]. We identified the SNPs passing the global significance line $\left(P<5.0 \times 10^{-8}\right)$ and the stringent significance threshold $\left(P<6.25 \times 10^{-9}\right)$, which is global significance divided by 8 because we used eight traits ( 2 dataset $\times 4$ time points) considering multiple testing burden. We considered the SNPs passing the stringent significance threshold as the significant SNPs and the SNPs which wasn't passing the stringent significance threshold, but passing global significance line as the suggestive SNPs. We downloaded the previously reported SNPs with "response to vaccine" from the GWAS catalog database on September 7, 2020 and compared our results with them. Next, we obtained the gene annotations of our SNPs with the SNPnexas tool [27] to examine the function of the annotated genes in the significant and suggestive SNPs. To select biologically important genes and their networks from the SNPannotated gene set, we used the STRING database version 11.0 to depict annotated gene networks [33], and extracted the genes with at least one link and their protein-protein interactions. 
(a)

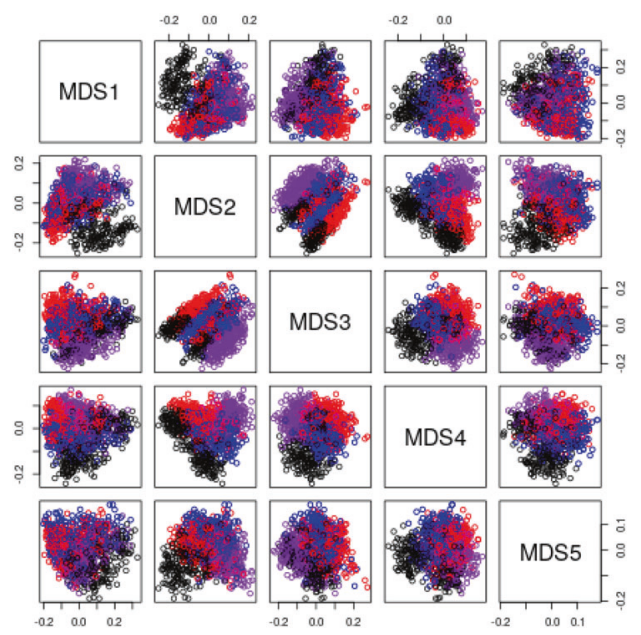

(b)

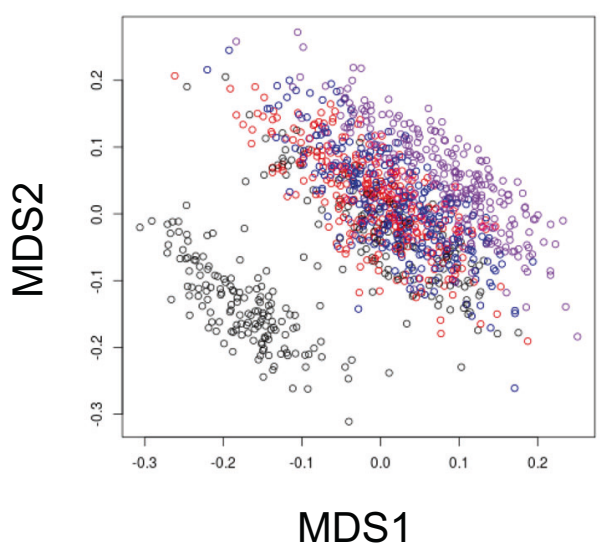

points colored red, blue, black, and purple represent Days $0,1,7$, and 90 samples, respectively

\section{Embedding the lymphocyte profiles to a Euclidean space by MDS}

\section{Comprehensive quantification of cell subset fractions}

Figure $\mathrm{S} 1$ shows the comprehensive change of the B cell subsets and T cell subsets of Days 1, 7, and 90 from Day 0 . The CD19-IgD-CD21-CD27+ subset was relatively increased after vaccination. That subset is considered to contain plasma cells. The $\mathrm{CD} 19+\mathrm{IgD}+\mathrm{CD} 21+\mathrm{CD} 27-$ subset was relatively decreased at the Day 7 profile and recovered at Day 90. That subset can be annotated as the naive $B$ cell subset. In the case of $\mathrm{T}$ cell FACS, the number of CD45RO-negative cells decreased, and the number of CD45RO-positive cells increased, which corresponds to the fact that the naive population differentiates into an effector/memory population. These results correspond to immunological knowledge. However, with this parametric model, it is difficult to comprehensively describe all the changes occurring after vaccination. Supplementary Files S3 and S4 show density plots of B cell FACS data and T cell FACS data for Person ID 1, and the cutoff value for each marker as an example of representative data. Also, the median value of each lymphocyte subset among 301 individuals at each time point is shown in Figs. S2 and S3. The box whisker diagram of all B cell subsets and $\mathrm{T}$ cell subsets is shown in Supplementary Files S5 and S6. Note that the quantification results of rare subsets are subject to automatic preprocessing, and quantification artifacts, such as dead cells are not removed.
All cytometry data from B cell FACS and T cell FACS were embedded in a low-dimensional Euclidean space that best reflected their dissimilarity. We call the MDS coordinates with the $i$ th largest eigenvalue in B cell FACS dataset and T cell FACS dataset as B_MDSi and T_MDSi, respectively. Figure S4 shows a plot of the top eigenvalues in B cell FACS and T cell FACS. From the eigenvalue plot, up to MDS5 of B cell FACS and up to MDS2 of T cell FACS were adopted as significant eigenvalues. They were used for the subsequent analysis as meaningful coordinates. Figure 2 shows a co-plot of the coordinates of 1173 samples in B cell FACS and T cell FACS. In the case of both B cell FACS and $\mathrm{T}$ cell FACS, the samples at different time points are separated on the MDS coordinates. Time-series information is consistently separated as in past studies [6-8]. It was confirmed that the MDS coordinate value is appropriate as a representative variable of the lymphocyte profile. And because the batch effect of cytometry data (Groups A and B) affects the MDS coordinate values (Fig. S5), we decided to consider this batch effect in the following GWAS analysis. In addition, it was suggested that multiple MDS coordinates not only explain time-series information, but also include other genetic or environmental factors that cause individual differences in the coordinate values.

Table $\mathrm{S} 1$ has the correlation coefficient and $P$ value of all paired MDS coordinates and lymphocyte subset fractions quantified with the parametric models, and Table S2 shows the 28 pairs with |correlation coefficient $>0.25$ and 
(a)

Density plot of $P$ value (T cell, Day 0 )

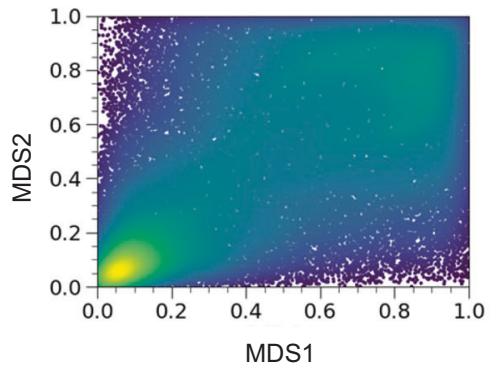

(b)

Density plot of $P$ value (B cell, Day0)

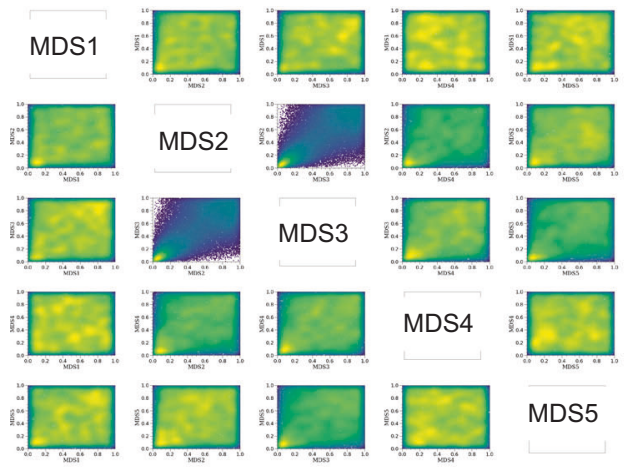

Fig. 3 Genetic features of $\mathrm{T}$ cell and $\mathrm{B}$ cell profiles derived from the GWAS. a Density plot of the $P$ values of T_MDS1 and T_MDS2 2 (Day0). b Density plot of the $P$ values of B_MDS1, B_MDS2,

Bonferroni-corrected $P<0.05$ that were extracted. The explanation of each MDS coordinate is written in Supplementary File S7. This result provides clues associating a subset of lymphocytes that interpret the meaning of each MDS coordinate.

\section{GWAS for MDS coordinates}

\section{Genetic features of the individual differences in human lymphocyte profiles}

To examine the genetic effects of the lymphocyte profile, a GWAS was performed on MDS coordinate values, and SNPs that were significantly related to these feature statistics were examined. The target traits are the coordinate values of MDS1, MDS2, MDS3, MDS4, and MDS5 in B cell FACS, and MDS1 and MDS2 in T cell FACS at Days $0,1,7$, and 90 , respectively. While the GWAS for Day 0 identified SNPs associated with steady-state lymphocyte profiles, the GWASs for Days 1, 7, and 90 identified SNPs associated with individual differences in immune responses at each time point after vaccination. We searched the literature for these SNPs. The QQ plots for Days 0, 1, 7, and 90 on each MDS coordinate are shown in Fig. S6.

Population structuring was considered to have little effect on GWAS results, because the genomic inflation factors were almost all 1 in all analyses (ranged from 1 to 1.01426).

Figure 3a shows a density plot of $P$ values for $\mathrm{T}$ cell MDS coordinates at Day 0. SNPs were observed at high densities in both MDS coordinates and regions with low $P$ values. In the same plot showing the result of T cell FACS data at other day points and B cell FACS data, a similar trend was observed for most MDS coordinate pairs (Fig. 3b and Figs. S7-S10). Figure S11 also shows a density plot of the values of the regression coefficients of all the MDS coordinates (B_MDS1, B_MDS2, B_MDS3, B_MDS4, B_MDS5, T_MDS1, and T_MDS2) on Day 0. Interestingly, the pair of B_MDS2 and B_MDS3 showed the largest correlation coefficient of the regression at 0.82 , while the correlation coefficient of these coordinate values was 0.16. In the other day points, the beta values of B_MDS2 and B_MDS3 showed a high correlation (Figs. S12-S14). 
From these results, we found that the SNPs associated with lymphocyte profiles were associated with multiple MDS coordinates. These results are valid because the differences in lymphocyte profiles were quantified as Euclidean distances on the MDS coordinate system, and some MDS coordinate values at the specific day points were correlated. The beta values of B_MDS2 and B_MDS3 showed a very high correlation coefficient overall, although there was also some correlation between those coordinate values. This suggests that B_MDS2 and B_MDS3 may be affected by common genetic effects.

After the integration based on the maximum $P$ value, we generated histograms and QQ plots with integrated $P$ values of T cell FACS and B cell FACS, as shown in Fig. 3c, d, respectively. The histograms show that the distribution of post-integration $P$ values using the maximum $P$ value takes the form of a mixture distribution of the uniform distribution from the SNP sets, which is irrelevant to the lymphocyte profile and the other distributions from the SNP sets that are associated with MDS coordinate space in all cases. The QQ plots show that the $P$ value after integration deviated from the uniform distribution in all cases. These deviations suggested that a large number of SNPs had a small effect on the differences in the lymphocyte profiles. It is considered that this is a general feature in the genetics of vaccination response, since QQ plots with similar characteristics were obtained in a past GWAS that used cytokine amounts as a trait [3]. This feature is common to the B cell profile and the $\mathrm{T}$ cell profile. However, the QQ plot on the $\mathrm{T}$ cell on Day 0 was especially deviated from the uniform distribution, and these weren't shown in the QQ plot of the B cell profile. The $\mathrm{T}$ cell profile in the steady state is affected more strongly by the SNPs, but the genetic effect on the $\mathrm{T}$ cell profile after vaccine intervention may be reduced.

\section{Candidate SNPs or genes explaining the individual differences in human lymphocyte profiles}

SNP level functional annotation in our GWAS identified seven significant SNPs $\left(P<6.25 \times 10^{-9}\right)$ and the 36 suggestive SNPs $\left(6.25 \times 10^{-9}<P<5.0 \times 10^{-8}\right)$ associated with either trait. The Manhattan plots are shown in Figs. S15 and S16. Table 1 shows the total 43 significant and suggestive SNPs, which we focused on for further analysis. We also searched for the SNPs that have been reported with the trait "response to vaccine" in the GWAS catalog database [34]. A total of 190 SNPs identified in 24 previous studies are registered (the output of the GWAS catalog is shown in Table S3). Although none of the SNPs identified in this research were included in these 190 SNPs, one annotated gene is common $(L P P)$. This gene has been reported to be a candidate gene in a GWAS study of cytokine responses to a smallpox vaccine [3].

In addition, rs6568431 has been reported to be associated with systemic lupus erythematosus (SLE) in multiple studies [35-37], and it has an $\mathrm{A}>\mathrm{C}$ allele and is situated in the intronic region of ATG5. ATG5 is a gene that plays a major role in autophagy and is also strongly associated with SLE $[38,39]$. The GWAS beta values of the A allele of rs6568431 for GWAS T_MDS1_day0 and T_MDS2_day0 of (ATG5) are 0.02 and -0.02 , respectively. This suggests that this SNP causes T_MDS1 drifts in the positive direction and T_MDS2 drifts in the negative direction on the $\mathrm{T}$ cell MDS space. Table S2 shows that $\mathrm{T}_{-}$MDS1 has a positive correlation with the fraction of $\mathrm{CD} 4+\mathrm{CD} 8$ $-\mathrm{CD} 45 \mathrm{RA}+\mathrm{CD} 45 \mathrm{RO}-\mathrm{CD} 25-\mathrm{CCR} 7+$ (annotated to CD4 + naive $\mathrm{T}$ cells), and T_MDS2 has a negative correlation with this subset fraction. In fact, the genotype of this SNP (AA, AC, or CC) is related to the abundance of this subset where the Jonckheere-Terpstra test $P=0.0024$ using the R package's clinfun's jonckheere.test function with the number of permutations set to 10,000 [40]. A box whisker diagram of each genotype is shown in Fig. S17. This SNP may be associated with SLE through individual differences in $\mathrm{T}$ lymphocyte profiles, in particular the CD4+ naive $\mathrm{T}$ cell subset.

In addition, we searched for our SNPs in our previous eQTL study using the same genotype data [26], the blood eQTL browser [41], and the eQTL database of the GTEx Consortium [42]. While none of the SNPs were common between our previous eQTL study and this study, a total of 14 out of 43 SNPs were found in the other two previous studies (Table S4). Given that many organs are involved in the immune response, the SNPs identified in this study may influence individual differences in lymphocyte profiles through the expression of these genes.

Next, we obtained the gene annotations of our SNPs with the SNPnexas tool to examine the function of the annotated genes in the GWAS. Table S5 shows the gene annotations of all 43 SNPs as the output of SNPnexas. To select biologically important genes and their biological links, we used the STRING database to depict annotated gene networks. Figure 4 shows the network of 12 genes with at least one link (ITPR1, OPN3, DNER, CYCS, ATG5, OSBPL3, MBP, PRKCB, CHML, ARHGAP26, KCNMA1, and EVAlA). The gene that is connected to the largest number of genes in the network is ITPR1. rs4685806 is situated in the intronic region of this gene. The CYCS gene is annotated to rs39426 with the second lowest GWAS $P$ value, which is situated $53 \mathrm{kbp}$ downstream of the end of this gene. This gene has been reported to be associated with SLE in a previous GWAS [37]. The PRKCB gene has a role in both oxidative stress induced autophagy and B cell activation [43, 44]. rs169140 is situated $13 \mathrm{kbp}$ upstream of the end of this 
Table 1 SNPs that passed the global significance line in B or T cell FACS

\begin{tabular}{|c|c|c|c|c|c|c|}
\hline rs ID & Chromosome & Position & REF allele & ALT allele & $P$ values & Trait \\
\hline rs 10460510 & 2 & $75,697,424$ & G & A & $2.10 \mathrm{E}-10 *$ & T_day0 \\
\hline rs39426 & 7 & $25,106,296$ & G & A & $7.97 \mathrm{E}-10^{*}$ & T_day90 \\
\hline rs 75504175 & 3 & $118,997,705$ & G & A & $2.18 \mathrm{E}-09 *$ & B_day7 \\
\hline rs3794852 & 18 & $74,709,289$ & $\mathrm{C}$ & $\mathrm{T}$ & $2.68 \mathrm{E}-09 *$ & T_day0 \\
\hline rs59422776 & 17 & 766,399 & $\mathrm{C}$ & $\mathrm{T}$ & $4.42 \mathrm{E}-09 *$ & T_day0 \\
\hline rs118160548 & 17 & 771,456 & A & G & $4.42 \mathrm{E}-09^{*}$ & T_day0 \\
\hline rs9919764 & 12 & $13,492,704$ & $\mathrm{C}$ & A & $4.47 \mathrm{E}-09 *$ & T_day7 \\
\hline rs 12611599 & 2 & $208,961,028$ & $\mathrm{C}$ & $\mathrm{T}$ & $7.73 \mathrm{E}-09$ & B_day7 \\
\hline rs 12614989 & 2 & $208,961,158$ & $\mathrm{~T}$ & $\mathrm{C}$ & $7.73 \mathrm{E}-09$ & B_day7 \\
\hline rs60057223 & 10 & $8,536,674$ & A & $\mathrm{C}$ & $1.05 \mathrm{E}-08$ & T_day0 \\
\hline rs11592991 & 10 & $8,539,331$ & G & A & $1.05 \mathrm{E}-08$ & T_day0 \\
\hline rs 10905390 & 10 & $8,537,695$ & A & $\mathrm{G}$ & $1.20 \mathrm{E}-08$ & T_day0 \\
\hline rs 10905391 & 10 & $8,540,328$ & $\mathrm{G}$ & $\mathrm{A}$ & $1.20 \mathrm{E}-08$ & T_day0 \\
\hline rs2696860 & 16 & $86,327,721$ & $\mathrm{~A}$ & G & $1.41 \mathrm{E}-08$ & B_day0 \\
\hline rs6568431 & 6 & $106,588,806$ & A & $\mathrm{C}$ & $1.45 \mathrm{E}-08$ & T_day0 \\
\hline rs6797423 & 3 & $126,215,130$ & $\mathrm{G}$ & $\mathrm{A}$ & $1.55 \mathrm{E}-08$ & T_day0 \\
\hline rs9381968 & 6 & $13,353,734$ & $\mathrm{~T}$ & $\mathrm{C}$ & $1.64 \mathrm{E}-08$ & T_day0 \\
\hline rs9530814 & 13 & $79,285,728$ & $\mathrm{~T}$ & $\mathrm{C}$ & $1.82 \mathrm{E}-08$ & B_day0 \\
\hline rs 169140 & 16 & $24,245,090$ & A & G & $2.00 \mathrm{E}-08$ & T_day0 \\
\hline rs6736713 & 2 & $230,366,542$ & $\mathrm{C}$ & $\mathrm{T}$ & $2.22 \mathrm{E}-08$ & T_day0 \\
\hline rs 138332350 & 1 & $241,794,224$ & $\mathrm{~T}$ & $\mathrm{C}$ & $2.39 \mathrm{E}-08$ & T_day0 \\
\hline rs78509568 & 2 & $197,398,200$ & $\mathrm{~T}$ & G & $2.43 \mathrm{E}-08$ & B_day90 \\
\hline rs4587178 & 6 & $98,421,991$ & $\mathrm{~T}$ & $\mathrm{C}$ & $2.47 \mathrm{E}-08$ & T_day0 \\
\hline rs78760834 & 17 & 740,734 & G & $\mathrm{C}$ & $2.50 \mathrm{E}-08$ & T_day0 \\
\hline rs58014646 & 20 & $41,344,189$ & A & $\mathrm{C}$ & $2.61 \mathrm{E}-08$ & B_day0 \\
\hline rs4668882 & 2 & $15,334,251$ & G & A & $2.83 \mathrm{E}-08$ & B_day7 \\
\hline rs55914228 & 10 & $126,896,675$ & A & G & $2.87 \mathrm{E}-08$ & T_day90 \\
\hline rs7069729 & 10 & $126,896,989$ & A & G & $2.87 \mathrm{E}-08$ & T_day90 \\
\hline rs6801602 & 3 & $188,519,594$ & A & G & $3.00 \mathrm{E}-08$ & T_day0 \\
\hline rs8073989 & 17 & $15,194,724$ & $\mathrm{~T}$ & $\mathrm{C}$ & $3.08 \mathrm{E}-08$ & T_day1 \\
\hline rs2051344 & 18 & $74,715,653$ & G & $\mathrm{T}$ & $3.13 \mathrm{E}-08$ & T_day0 \\
\hline rs4685806 & 3 & $4,772,692$ & $\mathrm{~T}$ & $\mathrm{C}$ & $3.47 \mathrm{E}-08$ & T_day0 \\
\hline rs35806 & 10 & $79,165,830$ & G & A & $3.48 \mathrm{E}-08$ & B_day7 \\
\hline rs 10436922 & 1 & $91,317,700$ & G & A & $3.65 \mathrm{E}-08$ & T_day0 \\
\hline rs1040893 & 6 & $106,596,087$ & $\mathrm{~T}$ & $\mathrm{C}$ & $4.07 \mathrm{E}-08$ & T_day0 \\
\hline rs76280036 & 5 & $142,276,784$ & $\mathrm{G}$ & $\mathrm{A}$ & $4.10 \mathrm{E}-08$ & B_day7 \\
\hline rs 13344319 & 19 & $54,921,227$ & $\mathrm{G}$ & A & $4.17 \mathrm{E}-08$ & T_day0 \\
\hline rs76425237 & 12 & $130,844,567$ & $\mathrm{~T}$ & $\mathrm{C}$ & $4.35 \mathrm{E}-08$ & B_day1 \\
\hline rs11920819 & 3 & $80,269,036$ & G & A & $4.61 \mathrm{E}-08$ & T_day90 \\
\hline rs7427090 & 3 & $80,274,761$ & $\mathrm{C}$ & $\mathrm{T}$ & $4.61 \mathrm{E}-08$ & T_day 90 \\
\hline rs4932564 & 15 & $92,176,277$ & A & G & $4.81 \mathrm{E}-08$ & T_day0 \\
\hline rs7639948 & 3 & $188,546,497$ & $\mathrm{~T}$ & $\mathrm{C}$ & $4.95 \mathrm{E}-08$ & T_day0 \\
\hline rs9851822 & 3 & $150,364,364$ & $\mathrm{G}$ & $\mathrm{A}$ & $4.98 \mathrm{E}-08$ & B_day90 \\
\hline
\end{tabular}

These were SNPs associated with individual differences of lymphocyte profiles (Day 0) and their change after vaccination (other than Day 0). Each column represents the following; rs ID of SNP, chromosome, position, reference allele, alternative allele, $P$ value of GWAS, B cell FACS/T cell FACS, and Day. $P$ values with asterisk $(*)$ indicates it passed the stringent significance threshold $\left(<6.25 \times 10^{-9}\right)$ 


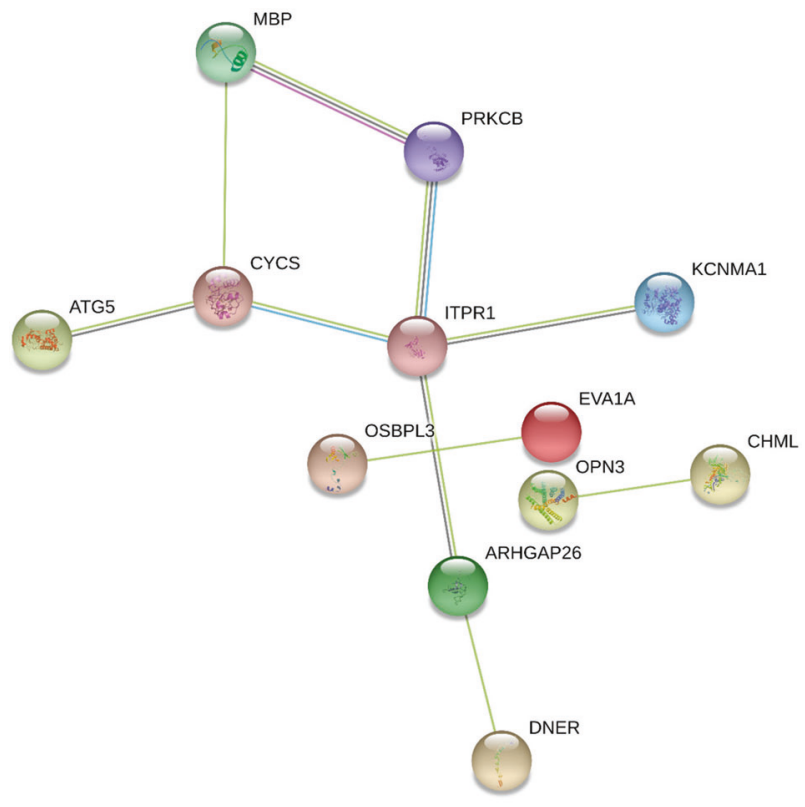

Fig. 4 Protein-protein networks of GWAS genes with at least one interaction in the STRING database. The color of the edge represents the type of interaction as defined in the STRING database. (Black: coexpression; purple: experimentally determined interaction; light blue: database annotated; and yellow: automated text mining). Genome-wide association study of individual differences of human lymphocyte profiles using large-scale cytometry data

gene. The EVAlA gene, annotated to rs 10460510 with the lowest GWAS $P$ value, is situated in the intronic region of this gene. This gene is reported to mediate both autophagy and apoptosis [45, 46]. A series of results suggests that mechanisms related to autophagy and SLE are associated with individual differences in lymphocyte profiles and their change after vaccination.

\section{Discussion}

When the phenotype takes the form of a point cloud from a distribution, such as a FACS result, it is difficult to analyze it with other variables. To analyze these distributions with regular variables with conventional statistical methods, the distribution should be expressed as a vector. In this study, using inter-distribution divergence and MDS, we extracted the independent feature statistics that best explained the differences in the overall lymphocyte profile. Lymphocyte profiles change dynamically with vaccination, and are difficult to describe completely. In addition, a small subset of lymphocytes has been suggested to play an important role in the immune system. It is also difficult to fit a parametric model, such as a bimodal mixture normal distribution model, with some markers. The MDS coordinate values are however effective data-driven and non-parametric feature statistics of the whole lymphocyte profiles.
The candidate genes we report in this study include those that have been reported in the literature to be related to immune phenotypes. We thus considered that we can identify novel candidate genes for individual lymphocyte profile differences and their changes after vaccination that previous GWASs, using conventional immune response biomarkers, such as titers and cytokines could not detect. Recently, personalized medicine, which takes into account such individual differences, has attracted increasing attention in regard to viral immune response or vaccine safety [47]. Unfortunately, the results of this study do not directly predict vaccination response or effectiveness, and are not sufficient to apply to personalized medicine. A detailed future study of the SNPs or annotated genes identified in this study may help to elucidate the molecular basis of individual differences in the immune response, as well as help to develop genomic markers to predict vaccination responses in individuals. In addition, interest in individual differences in response to viral infection has increased due to the COVID19 pandemic. The genetics of individual differences in response to vaccination as identified here could be a meaningful basis for further study.

In this study, using data-driven extraction of lymphocyte profile feature statistics, we combined cytometry data with SNP genotype data by positioning the cytometry data as one layer of a multi-omics approach. Multi-omics analysis combining multiple omics resources has become a highly useful approach and has successfully revealed various biological phenomena [48]. Our approach has enabled us to integrate cytometry data into a multi-omics analysis, which can contribute to the understanding of a complex biological system.

This study has some limitations when interpreting the results biologically. First, this study used relatively few samples. While this study and past GWAS QQ plots suggest the small involvement of many genes in the vaccine response, only 43 SNPs could be detected in our GWAS. We consider that the small sample size caused relatively large $P$ values considering the load of multiple testing due to conducting GWAS for eight traits ( $\mathrm{B} / \mathrm{T}$ cell profiles by four time points). This is likely the reason why only one gene $(L P P)$ was common between our GWAS and the previously reported genes in the GWAS catalog. Our GWAS probably missed many other candidate SNPs and genes reported in past GWAS. Second, it is unclear what kinds of functional SNPs the workflow used in this study captured. A relatively small number of SNPs were common between those identified in this study and those in previously reported eQTL studies. The method of this study may tend to detect SNPs associated with differences in the overall distribution of protein expression levels that are the combinatorial phenomenon of multiple proteins in pathways. This means that the eQTL analysis did not seem to be able to capture the heterogeneity, because eQTL is a 
transcriptome analysis of individual genes using bulk cells as samples, so heterogeneity cannot be captured by eQTL analysis using bulk transcriptome data.

The following four points can be listed as improvements in the workflow used in this study. In this study, the MDS coordinate with the smallest eigenvalue was excluded from the analysis. Although this does not explain much of the difference in cell population profiles, such a coordinate is not necessarily immunologically meaningless. Second, it is difficult to consider the batch effect of cytometry data because those data are a point cloud distribution. In this regard, the workflow used in this study can be improved. Third, when the number of samples of cytometric data is limited, the detection power of GWAS becomes small. The development of methods to improve statistical power will be a major improvement. Finally, the workflow used in this study can be applied not only to cytometry data, but also to single-cell RNA-seq (scRNA-seq) data. In recent years, scRNA-seq has been used not only for immunology, but also in various other biological fields [49]. Since scRNAseq data have higher dimensions than cytometry data, it will be necessary to develop steps for selecting markers for application. Modifying this workflow for scRNA-seq is an issue we will address in the future.

In this study, we estimated the distribution of lymphocyte profiles in peripheral blood from FACS data and extracted feature statistics. With the GWAS, we were able to identify SNPs related to differences in lymphocyte profiles. The workflow of this study is considered to be a powerful approach to data-driven identification of biological factors involved in the complex biological phenomena and diseases based on cell population profiles.

\section{Data availability}

FACS data and SNP genotype data are available under the condition of collaboration, because they are the resources of on-going studies. Please contact us for details on their availability.

Acknowledgements This work was supported by a KAKENHI Grantin-Aid from the Japan Society for the Promotion of Science (JSPS; grant number JP19J14816), and Core Research for Evolutionary Science and Technology (CREST; grant numbers JPMJCR1502 and JPMJCR15G1), and AIP Challenge of the Japan Science and Technology Agency (JST). We thank all staff and subjects of Kyoto University Nagahama 0th Cohort Project for providing data. And we also thank Prof. James Cai, Texas A\&M University, for his helpful comments on the manuscript and Dr. Maiko Narahara for her contribution in the initial phase of the study.

\section{Compliance with ethical standards}

Conflict of interest The authors declare that they have no conflict of interest.
Publisher's note Springer Nature remains neutral with regard to jurisdictional claims in published maps and institutional affiliations.

Open Access This article is licensed under a Creative Commons Attribution 4.0 International License, which permits use, sharing, adaptation, distribution and reproduction in any medium or format, as long as you give appropriate credit to the original author(s) and the source, provide a link to the Creative Commons license, and indicate if changes were made. The images or other third party material in this article are included in the article's Creative Commons license, unless indicated otherwise in a credit line to the material. If material is not included in the article's Creative Commons license and your intended use is not permitted by statutory regulation or exceeds the permitted use, you will need to obtain permission directly from the copyright holder. To view a copy of this license, visit http://creativecommons. org/licenses/by/4.0/.

\section{References}

1. Hagan T, Pulendran B. Will systems biology deliver its promise and contribute to the development of new or improved vaccines? From data to understanding through systems biology. Cold Spring Harb Perspect Biol. 2018;10:a028894.

2. Marees AT, de Kluiver H, Stringer S, Vorspan F, Curis E, MarieClaire $\mathrm{C}$, et al. A tutorial on conducting genome-wide association studies: quality control and statistical analysis. Int $\mathrm{J}$ Methods Psychiatr Res. 2018;27:e1608.

3. Kennedy RB, Ovsyannikova IG, Pankratz VS, Haralambieva IH, Vierkant RA, Poland GA. Genome-wide analysis of polymorphisms associated with cytokine responses in smallpox vaccine recipients. Hum Genet. 2012;131:1403-1421.

4. O'Connor D, Png E, Khor CC, Snape MD, Hill AVS, van der Klis $\mathrm{F}$, et al. Common genetic variations associated with the persistence of immunity following childhood immunization. Cell Rep. 2019;27:3241-3253.

5. Alegria GC, Gazeau P, Hillion S, Da¥"¥ien CI, Cornec DYK. Could lymphocyte profiling be useful to diagnose systemic autoimmune diseases?. Clin Rev Allergy Immunol. 2017;53:219-236.

6. Tsang JS, Schwartzberg PL, Kotliarov Y, Biancotto A, Xie Z, Germain RN, et al. Global analyses of human immune variation reveal baseline predictors of postvaccination responses. Cell 2014;157:499-513.

7. Nakaya HI, Wrammert J, Lee EK, Racioppi L, Marie-Kunze S, Haining WN, et al. Systems biology of vaccination for seasonal influenza in humans. Nat Immunol. 2011;12:786.

8. Obermoser G, Presnell S, Domico K, Xu H, Wang Y, Anguiano $\mathrm{E}$, et al. Systems scale interactive exploration reveals quantitative and qualitative differences in response to influenza and pneumococcal vaccines. Immunity 2013;38:831-844.

9. Carter KM, Raich R, Finn WG, Hero AO III. Fine: Fisher information nonparametric embedding. IEEE Trans Pattern Anal Mach Intell. 2009;31:2093-2098.

10. Gingold JA, Coakley ES, Su J, Lee D-F, Lau Z, Zhou H, et al. Distribution Analyzer, a methodology for identifying and clustering outlier conditions from single-cell distributions, and its application to a Nanog reporter RNAi screen. BMC Bioinforma. 2015;16:225.

11. Nakamura N, Okada D, Setoh K, Kawaguchi T, Higasa K, Tabara Y, et al. LAVENDER: latent axes discovery from multiple cytometry samples with non-parametric divergence estimation and multidimensional scaling reconstruction. 2019. https://doi.org/10. 1101/673434. Accessed 18 June 2019.

12. Miyake M, Yamashiro K, Tabara Y, Suda K, Morooka S, Nakanishi $\mathrm{H}$, et al. Identification of myopia-associated WNT7B 
polymorphisms provides insights into the mechanism underlying the development of myopia. Nat Commun. 2015;6:6689.

13. Sanderson RD, Lalor P, Bernfield M. B lymphocytes express and lose syndecan at specific stages of differentiation. Cell Regul. 1989;1:27-35.

14. Piatosa B, Wolska-Kusnierz B, Pac M, Siewiera K, Galkowska E, Bernatowska E. B cell subsets in healthy children: reference values for evaluation of $\mathrm{B}$ cell maturation process in peripheral blood. Cytom Part B Clin Cytom. 2010;78:372-381.

15. Agematsu K, Hokibara S, Nagumo H, Komiyama A. CD27: a memory B-cell marker. Immunol Today. 2000;21:204-206.

16. Janeway C, Murphy KP, Travers P, Walport M. Janeway's immuno biology. New York: Garland Science; 2008.

17. Zhu J, Yamane H, Paul WE. Differentiation of effector CD4 T cell populations. Annu Rev Immunol. 2009;28:445-489.

18. Treiner E, Lantz O. CD1d-and MR1-restricted invariant T cells: of mice and men. Curr Opin Immunol. 2006;18:519-526.

19. Bender A, Kabelitz D. CD4- CD8- human T cells: phenotypic heterogeneity and activation requirements of freshly isolated "double-negative" T cells. Cell Immunol. 1990;128:542-554.

20. Fischer K, Voelkl S, Heymann J, Przybylski GK, Mondal K, Laumer $\mathrm{M}$, et al. Isolation and characterization of human antigenspecific TCR $\alpha \beta+$ CD4-CD8-double-negative regulatory $\mathrm{T}$ cells. Blood 2005;105:2828-2835.

21. Richards SJ, Jones RA, Roberts BE, Patel D, Scott CS. Relationships between 2H4 (CD45RA) and UCHL1 (CD45RO) expression by normal blood CD4+ CD8-, CD4- CD8+, CD4CD8dim+, CD3 + CD4- CD8 - and CD3- CD4- CD8- lymphocytes. Clin Exp Immunol. 1990;81:149-155.

22. Taams LS, Smith J, Rustin MH, Salmon M, Poulter LW, Akbar AN. Human anergic/suppressive CD4+ CD25+ T cells: a highly differentiated and apoptosis-prone population. Eur $\mathrm{J}$ Immunol. 2001;31:1122-31.

23. Stephens LA, Mottet C, Mason D, Powrie F. Human CD4+ $\mathrm{CD} 25+$ thymocytes and peripheral $\mathrm{T}$ cells have immune suppressive activity in vitro. Eur J Immunol. 2001;31:1247-1254.

24. Caton AJ, Cozzo C, Larkin J III, Lerman MA, Boesteanu A, Jordan MS. CD4+ CD25+ regulatory T cell selection. Ann N. Y Acad Sci. 2004;1029:101-114.

25. Caraux A, Klein B, Paiva B, Bret C, Schmitz A, Fuhler GM, et al. Circulating human B and plasma cells. Age-associated changes in counts and detailed characterization of circulating normal CD138and CD138+ plasma cells. Haematologica 2010;95:1016-1020.

26. Narahara M, Higasa K, Nakamura S, Tabara Y, Kawaguchi T, Ishii $\mathrm{M}$, et al. Large-scale East-Asian eQTL mapping reveals novel candidate genes for LD mapping and the genomic landscape of transcriptional effects of sequence variants. PLoS ONE. 2014;9:e100924.

27. Dayem Ullah AZ, Oscanoa J, Wang J, Nagano A, Lemoine NR, Chelala C. SNPnexus: assessing the functional relevance of genetic variation to facilitate the promise of precision medicine. Nucleic Acids Res. 2018;46:W109-W113.

28. Lin J. Divergence measures based on the Shannon entropy. IEEE Trans Inf Theory. 1991;37:145-151.

29. Purcell S, Neale B, Todd-Brown K, Thomas L, Ferreira MAR, Bender D, et al. PLINK: a tool set for whole-genome association and population-based linkage analyses. Am J Hum Genet. 2007;81:559-575.

30. Dewey M. \{metap\}: meta-analysis of significance values. $R$ package version 1.1. 2019;1-26. http://www.dewey.myzen.co.uk/ meta/meta.html. Accessed May 2020.

31. Yang J, Weedon MN, Purcell S, Lettre G, Estrada K, Willer CJ, et al. Genomic inflation factors under polygenic inheritance. Eur J Hum Genet. 2011;19:807-812.
32. Turner SD. qqman: an R package for visualizing GWAS results using QQ and manhattan plots. J Open Source Softw. 2018;3:731.

33. Szklarczyk D, Gable AL, Lyon D, Junge A, Wyder S, HuertaCepas J, et al. STRING v11: protein-protein association networks with increased coverage, supporting functional discovery in genome-wide experimental datasets. Nucleic Acids Res. 2019;47: D607-D613.

34. Welter $\mathrm{D}$, MacArthur $\mathrm{J}$, Morales $\mathrm{J}$, Burdett $\mathrm{T}$, Hall $\mathrm{P}$, Junkins H, et al. The NHGRI GWAS Catalog, a curated resource of SNP-trait associations. Nucleic Acids Res. 2013;42: D1001-D1006.

35. Bentham J, Morris DL, Graham DSC, Pinder CL, Tombleson P, Behrens TW, et al. Genetic association analyses implicate aberrant regulation of innate and adaptive immunity genes in the pathogenesis of systemic lupus erythematosus. Nat Genet. 2015;47:1457.

36. Morris DL, Sheng Y, Zhang Y, Wang Y-F, Zhu Z, Tombleson P, et al. Genome-wide association meta-analysis in Chinese and European individuals identifies ten new loci associated with systemic lupus erythematosus. Nat Genet. 2016;48:940.

37. Gateva V, Sandling JK, Hom G, Taylor KE, Chung SA, Sun X, et al. A large-scale replication study identifies TNIP1, PRDM1, JAZF1, UHRF1BP1 and IL10 as risk loci for systemic lupus erythematosus. Nat Genet. 2009;41:1228.

38. Pyo J-O, Yoo S-M, Ahn H-H, Nah J, Hong S-H, Kam T-I, et al. Overexpression of Atg5 in mice activates autophagy and extends lifespan. Nat Commun. 2013;4:1-9.

39. Pierdominici M, Vomero M, Barbati C, Colasanti T, Maselli A, Vacirca D, et al. Role of autophagy in immunity and autoimmunity, with a special focus on systemic lupus erythematosus. FASEB J. 2012;26:1400-1412.

40. Seshan, VE. "Package 'clinfun'.” R package clinfun. 2018. https:// cran.r-project.org/web/packages/clinfun/clinfun.pdf. Accessed 13 April 2018.

41. Westra HJ, Peters MJ, Esko T, Yaghootkar H, Schurmann C, Kettunen J, et al. Systematic identification of trans eQTLs as putative drivers of known disease associations. Nat Genet. 2013;45:1238-1243.

42. Consortium Gte, others. Genetic effects on gene expression across human tissues. Nature 2017;550:204-213.

43. Patergnani S, Marchi S, Rimessi A, Bonora M, Giorgi C, Mehta $\mathrm{KD}$, et al. $\mathrm{PRKCB} /$ protein kinase $\mathrm{C}$, beta and the mitochondrial axis as key regulators of autophagy. Autophagy 2013;9:1367-1385.

44. Lutzny G, Kocher T, Schmidt-Supprian M, Rudelius M, KleinHitpass L, Finch AJ, et al. Protein kinase c-\$ $\$$-dependent activation of NF- $\kappa \kappa \$ B$ in stromal cells is indispensable for the survival of chronic lymphocytic leukemia $B$ cells in vivo. Cancer Cell. 2013;23:77-92.

45. Li M, Lu G, Hu J, Shen X, Ju J, Gao Y, et al. EVA1A/TMEM166 regulates embryonic neurogenesis by autophagy. Stem Cell Rep. 2016;6:396-410.

46. Shen X, Kan S, Liu Z, Lu G, Zhang X, Chen Y, et al. EVA1A inhibits GBM cell proliferation by inducing autophagy and apoptosis. Exp Cell Res. 2017;352:130-138.

47. Du J, Cai Y, Chen Y, He Y, Tao C. Analysis of individual differences in vaccine pharmacovigilance using VAERS data and MedDRA system organ classes: a use case study with trivalent influenza vaccine. Biomed Inf Insights. 2017;9:1178222617700627.

48. Hasin Y, Seldin M, Lusis A. Multi-omics approaches to disease. Genome Biol. 2017;18:1-15.

49. Chen G, Ning B, Shi T. Single-cell RNA-seq technologies and related computational data analysis. Front Genet. 2019;10:317. 